\title{
Estrategia didáctica basada en cantos shipibos para recuperar la lengua Shipibo-Konibo en niños de Educación Inicial, Ucayali
}

\author{
Didactic strategy based on shipibos songs to recover the Shipibo-Konibo \\ language in intial education children, Ucayali
}

\author{
Ruth K. Mendivel Geronimo ${ }^{1, *}$, Jesús M. Arias Huánuco ${ }^{2}$, Rosario M. Aguilar Melgarejo ${ }^{2}$ \\ ${ }^{1}$ Universidad Nacional Intercultural de la Amazonia, ${ }^{2}$ Universidad Nacional de Huancavelica
}

\section{RESUMEN}

La investigación tuvo como objetivo determinar la influencia de los cantos como estrategia didáctica en la recuperación de la lengua Shipibo Konibo en los niños de 5 años de la Institución Educativa Inicial Virgen de Fátima de Manantay en Pucallpa en el año 2015. La investigación fue del tipo aplicada, nivel explicativo y se utilizo el método experimental. El estudio tuvo un tamaño de muestra de 45 niños de 5 años de edad, y para la medición de la variable se utilizó una ficha de observación. Los resultados sobre recuperación de la lengua Shipibo Konibo de acuerdo al pre test indican que el $46,67 \%$ de la muestra están en un nivel medio; luego 33,33 \% están en el nivel bajo, y solo el 20,00 \% están en un nivel alto; en el post test se tiene que el 66,67 $\%$ están en el nivel alto de recuperación, luego un $33,33 \%$ se encuentran en un nivel medio y ningún niño estuvo en la opción bajo. Se concluye que los cantos como estrategia didáctica ha influido favorable y significativamente en la recuperación de la lengua Shipibo Konibo en los niños de 5 años de la Institución Educativa Inicial Virgen de Fátima de Manantay; asimismo los cantos han influido favorable y significativamente en la recuperación de la identidad cultural; también se ha identificado su influencia como estrategia didáctica en la recuperación de la lengua materna.

Palabras claves: Cantos, estrategia didáctica, recuperación, lengua y shipibo konibo.

\begin{abstract}
The objective of the research was to determine the influence of the songs as a didactic strategy in the recovery of the Shipibo Konibo language in childrens of 5 years old of the Initial Educational Institution Virgen de Fátima de Manantay in Pucallpa in 2015. The investigation was of the applied type, explanatory level and the experimental method was used. The study had a sample size of 45 children of 5 years of age, and for the measurement of the variable an observation card was used. The results on retrieval of the Shipibo Konibo language according to the pretest indicate that $46,67 \%$ of the sample are at a medium level; then $33,33 \%$ are at the low level, and only $20,00 \%$ are at a high level; in the post test it has that $66,67 \%$ are in the high level of recovery, then 33,33 $\%$ are in a medium level and no child was in the low option. It is concluded that the songs as a didactic strategy has favorably and significantly influenced the recovery of the Shipibo Konibo language in the 5 year old children of the Initial Educational Institution Virgen de Fátima de Manantay; also the songs have favorably and significantly influenced the recovery of cultural identity; Its influence has also been identified as a didactic strategy in the recovery of the mother tongue.
\end{abstract}

Keywords: Songs, didactic strategy, recovery, language and Shipibo Conibo.

Historial del artículo:

Recibido, 21 de febrero 2016; aceptado, 15 de abril de 2016; disponible en línea, 15 de julio de 2016

* Lic. en Educación Inicial, Mg. en Administración de la Educación. Dr. en Educación. Profesor Auxiliar UNIA.

Correo: ruthk15_28@hotmail.com 


\section{INTRODUCCIÓN}

En la Institución Educativa Inicial Virgen de Fátima de Manantay, Pucallpa, en las aulas de 5 años, consideradas como aulas interculturales se encuentran niños y niñas de las culturas Shipibo Konibo, Ashaninka, Awajun y Mestizo; los niños y las niñas ya no quieren hablar en su lengua, por tal motivo la enseñanza y aprendizaje se ve obligado a castellanizar; incluso son los mismos padres de familias que prohíben hablar a sus hijos en sus lenguas originarias.

Los niños provienen de diferentes comunidades nativas de la región Ucayali, y sus padres ya no se identifican con sus vestimentas típicas y optan por la ropa mestiza, pero si hablan perfectamente en Shipibo.

Existen dos tipos de niños, los nacidos en la misma ciudad de Pucallpa y aquellos que provienen de diferentes comunidades. Los niños provenientes de las comunidades si hablan perfectamente en Shipibo, y los docentes carecen de conocimientos de su cultura y es el motivo que influye para impartir el conocimiento ajeno.

La investigación es de tipo aplicada - longitudinal; dado el espacio contextual y temporal. El problema de investigación fue, ¿̇Cómo influyen los cantos como estrategia en la recuperación de la lengua Shipibo Konibo en los niños de 5 años de la Institución Educativa Inicial Virgen de Fátima de Manantay, Pucallpa 2015?. El objetivo fue determinar la influencia de los cantos como estrategia en la recuperación de la lengua Shipibo Konibo en los niños de 5 años de la Institución Educativa Inicial Virgen de Fátima de Manantay, Pucallpa - 2015. La hipótesis planteada fue que, los cantos como estrategia influyen significativamente en la recuperación de la lengua Shipibo Konibo en los niños de 5 años de la Institución Educativa Inicial Virgen de Fátima de Manantay, Pucallpa - 2015.

La estrategia que se fundamenta en los cantos, sustenta que una canción es una composición musical para la voz humana (comúnmente acompañada por otros instrumentos musicales) y con letra. Típicamente es para un solo vocalista, aunque puede también ser para un dueto, trío, cuarteto y/o para más voces (música coral). Para Ajello "Las palabras de las canciones son tradicionalmente de versificación poética, aunque pueden ser versos religiosos de libre prosa" (Ajello, 2003).

Las canciones pueden ser ampliamente divididas de muchas maneras distintas, dependiendo del criterio usado. Una división es entre "Canciones artísticas", "canciones de música popular", y "canto folclórico". Otros métodos comunes de clasificación son de propósito; por estilo (baile, balada y lieder) o por tiempo de origen (renacimiento, contemporáneo, etc.).
Coloquialmente, aunque es incorrecto, la palabra canción es usada para referirse a cualquier composición musical, incluyendo aquellas sin canto (sin embargo, en los estilos musicales que son predominantemente vocales, una composición sin trozos cantados, es a menudo nombrada instrumental).

El problema de cómo diseñar las actividades de aprendizaje reside en crear las situaciones de aprendizaje adecuadas para que los esquemas de conocimiento, que contribuye el alumno, evolucionen en un sentido determinado.

Dichas situaciones deben ser desarrolladas atendiendo a los diversos tipos de contenidos. Es decir, según se trate de datos, conceptos, habilidades, destrezas - actitudes deberán considerarse situaciones de aprendizaje diferentes.

Aquí las características de cada tipo de contenido y que situaciones favorecen su aprendizaje:

a) Aprendizaje de contenidos conceptuales. Se refiere, tanto al aprendizaje de contenidos factuales (básicamente datos), como a los contenidos propiamente conceptuales (ideas y conceptos) que los estudiantes deben alcanzar en una etapa determinada de su formación.

b) Aprendizaje de contenidos procedimentales. Se refiere a aquel que incluye entre otras cosas, las reglas, las técnicas, los métodos, las destrezas o habilidades, las estrategias y los procedimientos un conjunto de acciones ordenadas y finalizadas, es decir, dirigidas a la consecución de un objetivo".

c) Aprendizaje de contenidos actitudinales. Aprendizaje actitudinal por persuasión, cuándo un mensaje es lo suficientemente persuasivo para modificar una actitud existente (Arnau, 1986).

Teorías sobre los valores. La postura psicoanalítica, según Freud de niños internalizamos la autoridad de nuestros padres constituyendo el superego que puede ser gratificador (sentimiento de orgullo por hacer el bien) y castigador (sentimiento de culpa por hacer el mal) interno, este último componente es lo que él llama conciencia. Para Suárez "La conciencia y el deseo del niño de ser como sus padres sirven para guiar la conducta cuando la autoridad de los padres disminuye" (Suárez, 1998).

La postura de la Teoría del Aprendizaje, en la percepción de Vroom "los valores se adquieren por la experiencia y por el reforzamiento positivo o negativo (por ejemplo la tendencia del niño a imitar acciones). Se aprenden, por lo general, mediante la instrucción verbal" (Vroom, 1964).

La interpretación perceptual, para López "los valores se desarrollan en virtud de la retroalimentación que 
proporciona la propia actividad y guían la conducta" (López, 2005). O sea los valores se desarrollan cuando se tiene libertad de elección y se producen cambios como efecto de la propia conducta. Los adolescentes que perciben la relación entre su propia conducta y los resultados de esa conducta tienen un conjunto de valores más desarrollados.

\section{Cultura Shipibo Konibo}

Los Shipibo-Konibo hablan una lengua de la familia Pano, como idioma principal el Shipibo. Los pueblos Panos se suelen denominar por el nombre de algún animal con el que se identifican a sí mismos.

Según López y Küper (1999) desde las tradiciones ancestrales "los Shipibo se autodenominaron por sus eventos sucesivos de "Ani Xeati" o la gran fiesta, en donde, tanto varones como mujeres, después de varias semanas de masateada, en sus barbas y/o vigotes, se quedaban los afrechos o restos de masatos (especialmente en los de los hombres) que les hacía parecer como "shipibo" o mono Pichico (mono con barba blanca). Así shipi- designa al 'mono pichico'. Cuando personas de otras etnias les veían, se mofaban y se reían de ellos tildando de "monos pichicos"; se dice que así iniciaban las guerras, batallas campales".

A los Konibo, les gustaba comer la especie de anguila que se llama macana, que en lengua Shipibo se le dice: koni- designa a la especie de 'anguila'.

Mientras que los Xetebo, se le atribuye ésta autodenominación, dizque por que le gustaban comer alimentos malogrados o pescados "Huañohuaño", Así Xete, significa Rinahui, especie de buitre, ave carroñero. La terminología bo es el morfema de plural. Para López y Küper (1999) "el pueblo Shipibo-Konibo es uno de los pueblos indígenas del oriente peruano, cuya lengua pertenece a la familia lingüística Pano. Viven en los márgenes del río Ucayali y sus afluentes: Pisqui, Calleria, Tamaya y Aguaytia; y a las orillas de los lagos Imiria y Yarina. Y un grupo minoritario en el río Pachitea. El rio Ucayali es un río grande originario de la Cordillera de los Andes que se une el río Amazonas en el norte de Perú".

Se considera que río abajo de Pucallpa está el territorio Shipibo y río arriba el Konibo, pero en realidad hay comunidades de ambos grupos en las dos zonas porque se han mezclado entre sí, los Xetebo que antiguamente vivían río abajo de Contamana ahora se encuentran integrados a los Shipibos.

Los Shipibo-Konibos son producto de una serie de fusiones culturales entre tres grupos que anteriormente eran distintos entre sí: los Shipibos, los Konibos y los Xetebos; que se autodenominaban como jonibo o jonikonbo, que significa "hombres" o "verdaderos hombres" respectivamente.

Según diversos estudios, estos grupos se fusionaron para formar el pueblo Shipibo-Konibo. Los Xetebo fueron diezmados por las epidemias y las luchas con tribus vecinas; a la larga se integraron a los Shipibos debido a la conquista y a los matrimonios mixtos. Los Konibo también perdieron su identidad debido a los matrimonios mixtos con los Shipibos, y ahora se les considera como un solo grupo étnico.

Vida en común y grupos de familia múltiple es la forma tradicional en que los Shipibos han vivido durante muchas generaciones. En el pasado, los tres grupos de los Shipibo, Konibo, y Xetebo se consideraron comunidades separadas. Hoy en día, se mezclan en un grupo después de años de matrimonio. Están relacionados con la cultura y de la misma familia lingüística, el Pano. Ellos son los más comúnmente conocida como el Shipibo, o Shipibo Konibo.

Este pueblo ribereño se encontraba tradicionalmente asentado en las riberas del río Ucayali y sus afluentes. Actualmente, las comunidades shipibo-konibo se ubican en los departamentos de Ucayali, Madre de Dios (un número pequeño vive en el departamento de Madre de Dios a orillas del río Inambari), Loreto y Huánuco. Según el INEI para el 2007, la población de las comunidades auto identificadas como ShipiboKonibo se estimaba en 22517 habitantes, repartidas en más de 140 caseríos o comunidades nativas; siendo uno de los pueblos más numerosos de la Amazonía peruana.

\section{MATERIAL Y MÉTODOS}

Según Sierra (2002), el tipo de estudio de la presente investigación fue aplicada, porque "en éstos estudios se deben determinar y definir previamente las variables, luego se formulan hipótesis, los mismos que deben probarse por métodos estadísticos, trabajándose con muestras representativas y llegando al final a conclusiones".

El nivel de investigación es el explicativo, "las investigaciones explicativas buscan especificar las propiedades importantes de los hechos y fenómenos que son sometidos a una experimentación de laboratorio o de campo" (Sierra, 2002).

En el presente trabajo de investigación se ha utilizado el método científico como método general. Según Sierra "El estudio del método científico es objeto de estudio de la epistemología. Asimismo, el significado de la palabra "método" ha variado. Ahora se le conoce como el conjunto de técnicas y procedimientos que le permiten al investigador realizar sus objetivos" (Sierra, 1995). Como método específico se ha utilizado 
el método experimental, por cuanto nos permitió establecer la eficacia de la estrategia didáctica. El diseño general viene a ser el Pre Experimental, con pre test y post test (Hernández, 2006).

\section{GE: $0_{1} \quad \mathrm{X} \quad \mathrm{O}_{2}$}

Donde:

$$
\begin{aligned}
& \text { GE: Grupo Experimental. } \\
& 0_{1}: \text { Pre Test } \\
& \mathrm{O}_{2} \text { : Post Test } \\
& \mathrm{X} \text { : Manipulación de la Variable Independiente. }
\end{aligned}
$$

La población estuvo conformada por 45 niños de 5 años de edad de la Institución Educativa Inicial Virgen de Fátima de Manantay, Pucallpa en el 2015. La muestra fue censal y estuvo conformada por los mismos 45 niños. La técnica utilizada fue la observación.

Los instrumentos usados en la presente investigación fueron las fichas de observación estructurada y no estructurada. Se calculó el coeficiente de confiabilidad de la ficha de observación igual a 0,925. El coeficiente de validez, se realizó mediante el juicio de experto el cual alcanzó el $90 \%$. Como quiera que para los ítems sea válido se necesita un completo acuerdo entre los jueces, según Oseda, se concluye que dicho instrumento de investigación es válido (Oseda, 2011).

\section{RESULTADOS}

\section{Análisis del Pre Test}

En base a la tabla 1 se puede deducir que, en el Grupo Experimental se puede apreciar que 21 niños que representa el 46,67\% están en el nivel medio de recuperación de la lengua Shipibo Konibo, luego 15

Tabla 1: Pre Test, Variable Dependiente: Recuperación de la lengua Shipibo Konibo.

\begin{tabular}{lcc}
\hline \multicolumn{1}{c}{ Categoría } & Frecuencia & Porcentaje \\
\hline Alto & 9 & 20,00 \\
Medio & 21 & 46,67 \\
Bajo & 15 & 33,33 \\
\hline Total & 45 & 100,00 \\
\hline
\end{tabular}

niños que representa el 33,33 \% están en el nivel bajo y solamente 9 niños que representa el 20,00 \% están en el nivel alto. Es decir la mayoría estuvo en el nivel medio, el cual no es muy favorable. A decir de López y Küper (6) el diagnóstico refleja que los niños en edad pre escolar y escolar no se identifican con la lengua Shipibo Konibo, principalmente por factores externos como pueden ser la institución educativa, los docentes y sus propios compañeros/amigos de clase.
En base a la tabla 2 se puede deducir que en el Grupo Experimental, específicamente en la dimensión Identidad cultural, se puede apreciar que 24 niños que

Tabla 2: Pre Test: Dimensión N 1: Identidad Cultural.

\begin{tabular}{lcc}
\hline \multicolumn{1}{c}{ Categoría } & Frecuencia & Porcentaje \\
\hline Alto & 6 & 13,33 \\
Medio & 24 & 53,33 \\
Bajo & 15 & 33,33 \\
\hline Total & 45 & 100,00 \\
\hline
\end{tabular}

representa el 53,33 \% están en el nivel medio, luego 15 niños que representa el 33,33 \% tenemos en el nivel bajo y solamente 6 niños que representa el 13,33 \% están en el nivel alto; o sea la gran mayoría de los niños evaluados en esta primera dimensión estuvieron en el nivel medio, el cual no es muy favorable; ya que como menciona López los niños indígenas poco a poco están perdiendo su identidad cultural, el cual se manifiesta abiertamente y preocupa (López, 2014).

Según la tabla 3, en la dimensión lengua materna, se puede apreciar que 21 niños que representa el

Tabla 3: Pre Test: Dimensión N²: Lengua materna.

\begin{tabular}{lcc}
\hline \multicolumn{1}{c}{ Categoría } & Frecuencia & Porcentaje \\
\hline Alto & 9 & 20,00 \\
Medio & 21 & 46,67 \\
Bajo & 15 & 33,33 \\
\hline Total & 45 & 100,00 \\
\hline
\end{tabular}

$46,67 \%$ están en el nivel medio, luego 15 niños que representa el 33,33 \% están en el nivel bajo y 9 niños que representa el 20,00 \% están en el nivel alto. Es decir, la mayoría de niños en esta dimensión estuvieron en la opción media, el cual no es muy favorable.

\section{Análisis del Post Test}

Según la tabla 4; se puede apreciar que 30 niños

Tabla 4: Post Test: Variable Dependiente: Recuperación de la lengua Shipibo Konibo.

\begin{tabular}{lcc}
\hline \multicolumn{1}{c}{ Categoría } & Frecuencia & Porcentaje \\
\hline Alto & 30 & 66,67 \\
Medio & 15 & 33,33 \\
Bajo & 0 & 0,00 \\
\hline Total & 45 & 100,00 \\
\hline
\end{tabular}

que representa el 66,67 \% están en el nivel alto de recuperación de la lengua Shipibo Konibo, luego 15 niños que representa el 33,33\% estuvieron en el nivel medio y ningún niño estuvo en la opción bajo; 
Tabla 5: Post Test: Dimensión N 1: Identidad Cultural.

\begin{tabular}{lcc}
\hline \multicolumn{1}{c}{ Categoría } & Frecuencia & Porcentaje \\
\hline Alto & 27 & 60,00 \\
Medio & 15 & 33,33 \\
Bajo & 3 & 6,67 \\
\hline Total & 45 & 100,00 \\
\hline
\end{tabular}

es decir gracias a la aplicación de la estrategia de cantos shipibos se ha recuperado la lengua Shipibo Konibo, con mucha razón Sotocuro menciona que la "recuperación de nuestra identidad cultural se fundamenta en una necesidad de integración y ayuda mutua permanente entre sus actores" (Sotocuro, 2012).

Tabla 6: Post Test: Dimensión N²: Lengua materna.

\begin{tabular}{lcc}
\hline \multicolumn{1}{c}{ Categoría } & Frecuencia & Porcentaje \\
\hline Alto & 30 & 66,67 \\
Medio & 15 & 33,33 \\
Bajo & 0 & 0,00 \\
\hline Total & 45 & 100,00 \\
\hline
\end{tabular}

De acuerdo a la tabla 5, en la dimensión 1 Identidad cultural, 27 niños que representa el 60,00 \% están en el nivel alto, luego 15 niños que representa el 33,33\% en el nivel medio y solamente 3 niños que representa el 6,67 \% están en el nivel bajo; es decir la gran mayoría de los niños evaluados en esta primera dimensión estuvieron en el nivel alto, el cual es muy favorable.

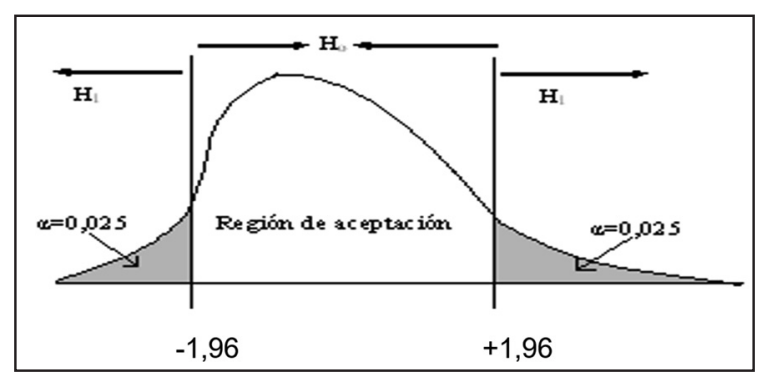

Figura 1: Región de aceptación y/o rechazo.

para este caso es la Prueba $Z$ y como en la hipótesis alterna $\left(\mathrm{H}_{1}\right)$ existe dos posibilidades $\left(H_{1}: \mu_{1}>\mu_{2}\right.$ ó $\mu_{1}<\mu_{2}$ ) se aplicó la prueba bilateral, 0 sea a dos colas.

\section{Cálculo del estadístico de prueba}

\section{Toma de decisiones}

Como -10,435<-1,96, entonces el estadístico calculado cae en la zona de rechazo, por lo tanto rechazamos la $\mathrm{Ho}$ y aceptamos la hipótesis alterna $\mathrm{H}_{1}$; con lo que concluimos que los cantos como estrategia didáctica han influido favorable y significativamente en la recuperación de la lengua Shipibo Konibo en los niños de 5 años de la Institución Educativa Inicial Virgen de Fátima de Manantay, Pucallpa en el 2015.

Cálculo del estadístico de prueba

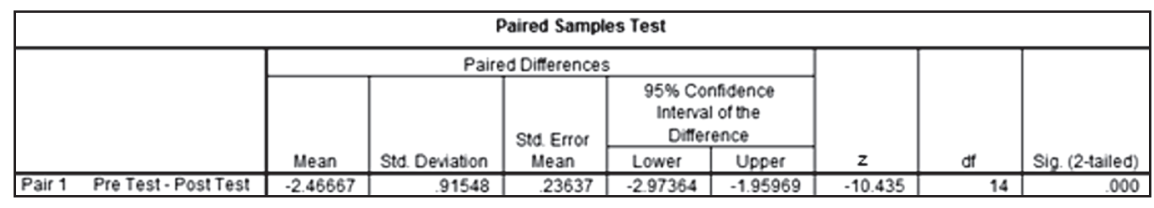

Según la tabla 6, en la dimensión 2 la lengua materna, se puede apreciar que 30 niños que representa el $66,67 \%$ están en el nivel alto, luego 15 niños que representa el 33,33 \% están en el nivel medio y ningún niño o niña estuvo en el nivel bajo. Es decir, la gran mayoría en esta dimensión estuvieron en el nivel alto, el cual es muy favorable.

\section{Prueba de Hipótesis}

$\mathrm{H}_{\mathrm{o}}$ : Los niveles de logro obtenidos en el post test y el pre test son iguales.

$\mathrm{H}_{1}$ : Los niveles de logro obtenidos en el post test y el pre test son diferentes.

El tipo de prueba: Es bilateral o a dos colas. El nivel de significancia utilizado en el diseño pre experimental es de: $\alpha=0,05$. El estadígrafo de Prueba más apropiado

\section{DISCUSIÓN}

Una interpretación adecuada, precisa y completa de los resultados que se han obtenido en la investigación, se inicia determinando los datos que obtuvieron antes de aplicar los cantos como estrategia en la recuperación de la identidad cultural en los niños de 5 años de la Institución Educativa Inicial Virgen de Fátima de Manantay, Pucallpa.

Empezamos comparando el problema o dificultad de la recuperación de la lengua Shipibo Konibo que presentan los niños de 5 años de edad de nuestra muestra en estudio con los problemas o dificultades que se han investigado en otros contextos, para encontrar si es que existen semejanzas o diferencias respecto a sus logros y dificultades. 
Comenzamos este apartado, manifestando que los datos obtenidos en el pre test acerca de los niveles de recuperación de la lengua Shipibo Konibo, nos evidencian que los integrantes de nuestra muestra presentan un nivel medio, tal como se muestra en las tablas del 1 al 6, donde obtuvieron en promedio las puntuaciones cualitativas "regulares o medias", ya que las mayorías de las frecuencias estaban en la opción "medio" que significa en "proceso", esto significa que dichos niños de 5 años a veces recuperan la lengua Shipibo Konibo. Asimismo, los niños de 5 años, en el pre test presentaban dificultades en reconocer muchas costumbres de su comunidad, no identificaban con facilidad su lengua, peor aún en la identificación y diferenciación de las ideas referidas a la identidad cultural.

Como consecuencia de ello se les hacía más difícil hacer inferencias, predicciones de las ideas que se encuentran en la recuperación de la lengua Shipibo Konibo.

Asimismo, como se puede apreciar se ha probado estadísticamente la hipótesis de estudio, ya que se ha demostrado que si existe diferencias significativas en los resultados del pre test y post test en la experimentación de la estrategia basada en los cantos. Y efectivamente como se puede evidenciar en la parte del análisis e interpretación de los resultados, la mencionada estrategia de cantos ha influido favorablemente en la recuperación de la lengua Shipibo Konibo de niños de 5 años de la Institución Educativa Inicial Virgen de Fátima de Manantay, Pucallpa.

Muestra de ello tenemos las diferencias marcadas de los niveles de logro en el pre test y post test gracias a la aplicación de la estrategia de cantos. Definitivamente los niños tienen una capacidad excepcional de crear y comunicarse a cada instante y cuando ello ocurre, se sienten realizados. Potenciemos esta inteligencia, ya que de ella depende la identidad cultural de los niños afirma Piaget (Piaget, 1959).

Por lo tanto, a decir de Zacarías "El ser humano desde la niñez tiene una gran facultad que es la comunicación, la utilización de su lenguaje que va desarrollándose en relación con la edad evolutiva; y esto debe ser aprovechada a lo máximo en todas sus esferas, principalmente en el desarrollo de su identidad cultural e identificación de su lengua materna" (Zacarias, 2015).

Dichas potencialidades deben aprovecharse en las instituciones educativas para mejorar la identidad cultural de los pueblos amazónicos.

Y para concluir, con la prueba $\dagger$ de Student, con un nivel de significancia del $5 \%$, se ha obtenido una $\dagger=$ -10,43; con lo que se rechaza la $\mathrm{H} 0$ y se acepta la $\mathrm{H} 1$, con lo cual se puede manifestar contundentemente que existe suficiente evidencia y criterio para concluir afirmando que la estrategia didáctica basada en cantos ha favorecido la recuperación de la lengua materna en los niños de 5 años de la Institución Educativa Inicial Virgen de Fátima de Manantay, Pucallpa, el cual contrastamos la hipótesis en todo su contenido y extensión.

Algunas conclusiones son:

Se ha determinado con un nivel de significancia del 5 $\%$ que los cantos como estrategia didáctica ha influido favorable y significativamente en la recuperación de la lengua Shipibo Konibo en los niños de 5 años de la Institución Educativa Inicial Virgen de Fátima de Manantay, Pucallpa - 2016.

Los cantos como estrategia didáctica han influido favorable y significativamente en la recuperación de la identidad cultural en los niños de 5 años de la Institución Educativa Inicial Virgen de Fátima de Manantay, Pucallpa - 2015.

Los cantos como estrategia didáctica han influido favorable y significativamente en la recuperación de la lengua materna en los niños de 5 años de la Institución Educativa Inicial Virgen de Fátima de Manantay, Pucallpa - 2015.

\section{REFERENCIAS BIBLIOGRÁFICAS}

Ajello, A. M. (2003). Manual de psicología de la educación. España: Popular.

Arnau, J. (1986). Psicología Experimental, un Enfoque Metodológico. México: Trillas.

Hernández , R., Fernández , C., \& Baptista, P. (2006). Metodología de la Investigación. 4. México: Mc Graw Hill.

López , L. E., \& Küper , W. (20/05/1999). La Educación Intercultural Bilingüe en América Latina: Balance y Perspectivas . Iberoamericana de Educación.

López, M. (2014). Educación Intercultural Bilingüe. $5^{\circ}$. Chimborazo, Ecuador: Retablo.

López, N. (2005). Tendencias Pedagógicas contemporáneas . 3ra. Lima - Perú: José Carlos Mariátegui.

Oseda, D. (2011). Metodología de la Investigación. $3^{\circ}$ . Huancayo: Pirámide.

Piaget, J. (1959). La formación del símbolo en el niño. México: Fondo de Cultura Económica.

Sierra, R. (1995). Técnicas de Investigación Social. Madrid: Paraninfo.

Sierra, R. (2002). Tesis Doctorales. Madrid: Paraninfo.

Sotocuro, A. (2012). Comunidades indígenas altoandinas y amazónicas en latinoamericana. $3^{\circ}$. 
Río Cuarto: Universidad de Campiñas. Suárez, P. (1998). Hacia una Pedagogía del Conocimiento. Santa Fé de Bogotá: Mc. Graw-Hill. Vroom, V. (1964). Work and motivation [Trabajo y motivación]. New York: Wiley.
Zacarías, C. M. (2015). La Cosmovisión Amazónica. $2^{\circ}$. Ucayali: UNIA. 